\title{
L'ERREUR DANS L'APPRENTISSAGE DE LA PRODUCTION ÉCRITE EN CLASSE DU FLE : REPÉRAGE, TRAITEMENT ET REMÉDIATION
}

\author{
Mounir BOURRAY
}

Université Moulay Ismaïl, Maroc

mounirbourray@gmail.com

Article reçu le 15 novembre 2016 | révisé depuis le 18 novembre 2016 | accepté le 30 décembre 2016

RÉSUMÉ. Au cycle secondaire qualifiant, l'apprentissage du français, langue étrangère (FLE), acquiert une importance particulière étant donné son rôle essentiel dans les études supérieures et par là-même à l'accès au marché du travail. Cet apprentissage demeure submergé des erreurs commises par les apprenants dans les différentes activités malgré le changement des programmes et l'application de nouvelles approches et démarches pédagogiques imposées par la nouvelle réforme de 1999. Ainsi, notre article, qui se donne pour objectif d'analyser et de traiter l'inventaire d'erreurs que nous avons recueilli lors d'une activité de production écrite réalisée en classe du FLE au cycle secondaire qualifiant, précisément en deuxième année baccalauréat, pendant l'année scolaire 2015 / 2016, constituera l'un des modèles auxquels pourront se référer les enseignants, notamment les futurs enseignants, pour améliorer leurs approches et leurs pratiques en classe.

Mots-clés : analyse, apprentissage, erreur, français langue étrangère (FLE), production écrite, remédiation.

\begin{abstract}
ABSTACT. In High school, teaching and learning French as a foreign language (FFL) acquires a paticular importance due to its primordial role in higher education and thus access to the professional world. This learning remains overwhelmed by the errors committed by the learners in different activities despite the change of the programs and the application of new approaches and the pedagogical improvements imposed by the reform of 1999. Our article, which aims to analyze and treat the inventory of errors we have collected during a written production activity carried out in the class of the FLE in high school, precisely in the second year baccalaureate, During the 2015/2016 school year, aims to produce a model that can be used by teachers, in particular future teachers, to improve their approaches and practices in the classroom.
\end{abstract}

Keywords: analysis, error, French as a foreign language (FFL), learning, remediation, writing. 


\section{INTRODUCTION}

Lors de l'apprentissage du français, la question du repérage, de l'analyse et du traitement des erreurs et des insuffisances reste très récurrente. En effet, les enseignants ainsi que leurs apprenants cherchent inlassablement des solutions pour faire face à toutes les situations - problèmes rencontrées. Pour ce faire, nous, formateurs, enseignants, parents d'élèves et responsables, pouvons développer, d'après Henri Frei, Stephen-Pit Corder et Rémy Porquier, deux comportements à l'égard des erreurs des apprenants. Pour le premier, si nous arrivions à fonder une approche pédagogique parfaite qui permettra un bon déroulement des apprentissages, il serait sûr et certain que les apprenants ne commettront ni d'erreurs ni de fautes pour acquérir de nouvelles compétences. Pour le deuxième, l'erreur est prise pour une partie intégrante $\mathrm{du}$ processus d'enseignement / apprentissage; donc, il est évident qu'elle apparaîtra à tout moment de ce processus malgré les efforts que nous pourrons fournir à ce stade.

S'inscrivant sous le cadre du deuxième comportement, notre étude, qui se donne pour objectif de repérer, d'analyser et de traiter de ces quelques erreurs marquant l'apprentissage du FLE, précisément la production écrite au cycle secondaire qualifiant, essaiera de rapporter des éléments de réponses aux questions suivantes : Qu'estce qu'une erreur en didactique des langues ? Quelles sont les différentes attitudes que nous pouvons adopter vis - à vis des erreurs commises par les apprenants au cours de l'apprentissage du FLE, précisément de la production écrite ? De quels types et de quelles natures sont- elles? Pourrons- nous élaborer un plan d'action pour mieux traiter les différents types d'erreurs collectées?

«L'erreur est humaine», nous a-t-on a appris depuis notre plus jeune âge. Et c'est peut être l'une des rares idées qu'on ne peut contester. Cependant, comment définit-on une erreur? Plusieurs définitions ont essayé d'expliquer cette notion. Commençons par la définition élaborée par Lennon (1991), et qui avance qu'une erreur est "une construction ou combinaison linguistique qui dans un contexte donné ne peut être produite par l'utilisateur maîtrisant une langue maternelle" (p.181).

Dans la même perspective, Caroline (2014) ajoute $\mathrm{qu}^{\prime}$ "au cours de l'apprentissage, les apprenants d'une langue étrangère commettent des erreurs; les élèves en classe d'immersion ne font pas exception. Certes, les erreurs sont indispensables à l'apprentissage, cependant il est nécessaire d'enseigner certaines stratégies de repérage et de correction afin qu'elles aient un effet constructif sur les productions écrites (et orales) des apprenants et que les compétences de ces-derniers se rapprochent de plus en plus de celles d'un natif". (p.13)

De ces deux définitions, nous pourrons en déduire que, dans le contexte de l'apprentissage d'une langue étrangère, l'erreur a toujours été considérée comme quelque chose de négatif qu'il fallait à tout prix éviter. D'une part, elle représentait un signe de l'inadéquation d'une méthode $d^{\prime}$ enseignement; d'autre part, on ne pouvait que se plier à la nature humaine et accepter l'erreur tout en essayant de trouver des moyens de la gérer. Cette idée a changé graduellement avec le temps puisqu'on s'est rendu compte qu'on pouvait apprendre de nos erreurs.

L'une des approches les plus connues à travers l'histoire concernant l'erreur est celle qui la considère comme un résultat négatif et qui doit même être sanctionnée et punie. Différentes sociétés ont pris l'habitude de représenter l'erreur comme un obstacle qui entrave le progrès. Punir l'erreur était une pratique allant de pair avec le processus de l'enseignement-apprentissage et représentait un instrument de pouvoir dans les stratégies d'enseignement. À cet égard, l'erreur était persécutée et le reste encore à nos jours malgré les différentes orientations qui interdisent ce genre de comportement. L'idée de l'erreur, comme un résultat qu'il fallait éviter, a été supportée par les behavioristes, vu qu'elle était considérée, dans ce sens, comme un obstacle à l'apprentissage des langues. Les behavioristes concevaient l'erreur comme une défaillance du système d'enseignement à laquelle il fallait remédier au travers d'un 
travail intensif sur les formes à proprement dites inadéquates.

Une conception assez différente du Behaviorisme et celle qui appuyait l'idée selon laquelle, il n'y a pas de progrès sans erreurs. L'erreur comme un élément de progrès, est une idée basée sur les propos de Chomsky selon lesquels l'enfant génère le langage et la langue à travers des structures universelles innées. Dans cette optique, en usant de ce code symbolique, l'enfant peut avoir accès à différents éléments du savoir, non à travers un processus d'enseignement mécanique, mais à travers un processus de construction mentale qui se base sur la pratique et l'erreur. L'idée se confirme chez les apprenants d'une seconde langue qui forment des structures hypothétiques à propos des règles de langue cible et qui les confirment au fur et à mesure avec leurs erreurs et expériences avec le système de cette dernière. C'est de cette manière que l'erreur permet l'évolution et le progrès dans l'apprentissage.

\section{MÉTHODE}

Cette étude que nous avons menée dans le cadre d'un travail sur les erreurs affectant l'apprentissage de la langue française comme langue étrangère est effectuée à l'enceinte de quatre lycées de la délégation de Meknès (le Nord du Maroc), à savoir El-Khaouarizmi, Ain Karma, Bnou ElBayter et Omar Bnou El-Khattab. Dans le tableau suivant, nous présentons les statistiques bien détaillées concernant chaque établissement:

Tableau 1. La situation des établissements de notre enquête

\begin{tabular}{|c|c|c|c|c|}
\hline Secteur & Etablissement & Filière & Nombre de classe & effectif \\
\hline & & Sciences Physiques & 1 & \\
\hline & El-Khaouarizmi & $\begin{array}{c}\text { Sciences de vie et } \\
\text { de terre }\end{array}$ & 2 & 370 \\
\hline & & Lettres & 1 & \\
\hline & & Sciences Humaines & 4 & \\
\hline & & Sciences Physiques & 1 & \\
\hline \multirow[t]{10}{*}{$\begin{array}{l}\text { Secteur } \\
\text { public }\end{array}$} & Bnou L-Baytar & $\begin{array}{c}\text { Sciences de vie et } \\
\text { de terre }\end{array}$ & 1 & 224 \\
\hline & & Sciences Humaines & 2 & \\
\hline & & Sciences Physiques & 2 & \\
\hline & Omar Bnou l-Khattab & $\begin{array}{c}\text { Sciences de vie et } \\
\text { de terre }\end{array}$ & 3 & 424 \\
\hline & & Sciences & 4 & \\
\hline & & Mathématiques & & \\
\hline & & Sciences Humaines & 2 & \\
\hline & & Sciences Physiques & 1 & \\
\hline & Ain Karma & $\begin{array}{c}\text { Sciences de vie et } \\
\text { de terre }\end{array}$ & 1 & 238 \\
\hline & & Sciences Humaines & 1 & \\
\hline Total & 4 & ------------------ & 26 & 1256 \\
\hline
\end{tabular}

Notre étude a ciblé les apprenants de la deuxième année baccalauréat avec ses différentes options scientifiques, littéraires et économiques. Appartenant à une tranche d'âge entre 17 et 21 ans, ces apprenants suivent effectivement le même programme composé de trois œuvres littéraires, à savoir Il était une fois un vieux couple heureux de Mohammed Khair-Eddine, de Candide ou l'optimisme de Voltaire et Le
Père Goriot d'Honoré de Balzac, à partir desquelles les enseignants peuvent élaborer les différentes activités du même projet séquentiel (lecture, langue, communication orale et production écrite). Cet apprentissage de la langue française se réalise en concurrence avec plusieurs langues maternelles et étrangères prises en charge par le champ scolaire des apprenants comme 
l'arabe, l'amazighe, l'anglais, l'espagnol et parfois l'allemand.

Notre choix de travailler sur la production écrite n'est pas arbitraire, mais il est justifié par le fait que cette activité occupe une place centrale dans le processus d'apprentissage en classe du FLE, particulièrement au secondaire qualifiant, dans la mesure où elle constitue le couronnement d'une thématique quelconque rassemblant tout ce que l'apprenant a acquis tout au long des différentes activités, notamment de lecture, de langue et de communication orale. En plus, elle permettra à l'enseignant et à ses apprenants de dégager toutes les erreurs marquant l'écrit, les répertorier, en déterminer les sources et la fréquence de leur apparition dans le texte afin de pouvoir, par la suite, remédier aux imperfections en mettant au point les dispositifs et les stratégies nécessaires.

A ce stade, notre choix s'est focalisé sur le type narratif pour deux raisons: premièrement, la majorité des apprenants de la deuxième année baccalauréat sont initiés au travail sur le texte narratif ou le récit, et, ce travail a commencé depuis leurs études à l'école primaire; et, deuxièmement, par ce type de texte, ils acquièrent de nouvelles compétences de communication narrative qui peuvent les aider à faire partager, par la suite, leurs propres expériences avec les autres en suivant un raisonnement logique.

Ainsi, le sujet d'écriture que nous avons proposé en classe se présente comme suit: "Vous retrouvez un objet de votre enfance (un jouet, un cahier de souvenir, un bracelet...)». Rapportez toutes les circonstances de la trouvaille en décrivant votre réaction et vos sentiments à cette occasion qui vous rappelle une période de votre passé». Les apprenants ont, donc, pour tâche de produire un récit autobiographique marqué par l'emploi de la première personne du singulier «je », des temps du récit et des indicateurs spatio-temporels pour garantir son organisation. Pour accomplir cette tâche, l'enseignant, de sa part, doit les inciter à insérer des descriptions.

Notre test sur l'écrit a été élaboré en deux étapes fondamentales. Dans la première étape, qui a correspondu au jour «J -2 », nous avons demandé à tous les enseignants du français qui ont accepté de collaborer à cette recherche de présenter à leurs apprenants dans une séance d'activité orale et sous forme d'exposé ou séminaire, le vocabulaire ainsi que les outils linguistiques et non-linguistiques nécessaires pour accomplir leur tâche rédactionnelle. Dans la deuxième étape, qui a coïncidé au jour «J», nous avons conçu pour cette activité rédactionnelle un temps de 60 minutes qui a débuté dès que l'enseignant écrivait le sujet sur le tableau accompagné des différentes consignes à suivre. Son travail ne se limitait pas à écrire le sujet sur le tableau, mais il le dépassait à faire un petit rappel aux apprenants des différentes étapes de la production écrite en indiquant le coup temporel réservé à chacune d'elles. Il est à noter que la réalisation de ce travail avait eu lieu le matin durant une séance ordinaire de la production écrite pour aider les apprenants à mieux se concentrer.

La collecte du corpus d'étude se réalise en deux temps. En premier temps, nous avons récupéré toutes les copies d'élèves et les avons réparties en :

- Copies recueillies : 1192

- Copies examinées : 886

- Copies non - examinées : 306

En deuxième temps, nous avons adopté deux méthodes pour étudier les erreurs. La première qui est purement quantitative consiste à repérer à partir des copies examinées toutes les erreurs qui affectent les compétences

grammaticales, morphologiques, sémantiques, syntaxiques, orthographiques et lexicales commises par les apprenants; et, la deuxième qui est interprétative consiste à décrire les erreurs véhiculées dans les différentes productions collectées afin de les traiter, d'en expliquer les sources et d'y suggérer les remédiations adéquates.

\section{RÉSULTATS ET DISCUSSION}

Nous avons opté, dans notre étude, pour la classification qui répartit les erreurs dans un texte en deux grandes catégories, erreurs de contenu qui sont issues de l'incompréhension des consignes et du manque d'organisation globale du texte, et 
erreurs de forme, qui affectent la langue avec tous ses aspects.

\section{Erreurs de contenu}

\section{Erreurs dues à l'incompréhension des consignes à suivre}

Avant même de commencer à rédiger leurs textes, les apprenants sont obligés de lire les consignes et les mieux comprendre afin de les appliquer. Cet acte n'est pas toujours évident pour eux vu qu'il pourrait être pris, de par sa complexité, pour une «agression mentale» exercée par leur enseignant. Les apprenants se trouvent, ainsi, dans une situation ambigüe devant laquelle ils ne savent ni quoi faire, ni comment le faire, ni quand ils le feront.

À cet égard, dans notre étude, nous avons mis l'accent sur deux insuffisances très essentielles. La première est dûe à l'incapacité de l'apprenant, d'une part, à donner le bon sens aux mots - clés constituant le sujet, et, d'autre part, à répondre correctement aux questions correspondant aux consignes avancées. Ce problème d'incompréhension l'induit automatiquement à produire des textes qui ne touchent pas évidemment l'essence du sujet proposé.

La deuxième met l'apprenant en face d'une difficulté aussi bien compliquée que la première. Il sait bien qu'il rédigera un texte narratif, mais au lieu de suivre le schéma narratif qui exige de présenter au début les personnages, le temps et les lieux, il préfère commencer par les actions et les aventures et présenter au fil de l'histoire les personnages et le cadre spatio-temporel. Cet ordre des faits adopté perturbe la majorité des apprenants et les pousse à commettre des erreurs d'organisation touchant le contenu de leurs écrits.

\section{Erreurs issues $d u \quad m a n q u e$ d'organisation du texte}

Quand nous avons choisi de travailler sur ce type d'erreur, nous étions conscient que cette tâche ne serait pas facile dans la mesure où elle touchera plusieurs niveaux de l'organisation globale des textes, à savoir :
- La progression thématique qui est absente dans la totalité des productions écrites mises à l'examen, surtout celles produites par les élèves des filières littéraires et économiques. Ils ne développent pas leurs récits en suivant une progression thématique qui assurera évidemment un enchaînement logique des événements de l'histoire, à l'exemple de la progression à thème constant, de la progression à thème éclaté ou de la progression linéaire). Ainsi, nous présentons deux extraits illustrant ce propos:

Passage (1):«L'objet été jauni et totalement déchirer. Je sorti heureux de ma chambre. Toutes la famille s'est endormi ce jour - là » Passage (2) : «En cherchant dans le placart de mes parents, j'ai trouvé mon ancien alboum. C'était presque l'heur de l'école, je l'ai raporter avec moi pour le montré à mes amis. Driss a passé derrière moi ; nous partions ensemble chaque jour à l'école »

Les deux passages ci-dessus n'adoptent aucun type de progression thématique vu qu'ils sont formés de phrases décousues. Chaque phrase développe un thème et un propos différents de la phrase qui la suit, à l'exemple des deux premières phrases du premier passage: la phrase (1) «L'objet (Thème 1) été jauni et totalement déchirer (Propos 1) » et « Je (Thème 2) sorti heureux de ma chambre (Propos 2)».

- La cohésion - Les apprenants doivent produire des textes qui gardent un certain raisonnement logique afin que le lecteur puisse les lire sans fournir trop d'effort. Et s'il y a une insuffisance à ce point - là de la part des lycéens, c'est dû essentiellement à l'absence de plusieurs éléments, comme la concordance entre les temps verbaux, les mots de reprise et de substitution (les pronoms, les groupes de mots et les synonymes) et les connecteurs logiques (en premier lieu, en deuxième lieu, d'abord, ensuite...), qui garantissent la clarté du message, la variété des idées et la fluidité des textes. Voici deux passages mettant au clair ce que nous venons d'avancer :

Passage 1: Ma mère m'a demandé de rammener le vase de la cave. En cherchant dans les placard, j'ai 
trouvé mon ancienne poupée pâlie et abîmé. Et j'avais peur parce que la cave était à moitié allumé...

Passage 2: Mes parents en sortant fire les corses m'ont demendé de mettr ma chambr en ordre. J'ai terminer mes devoir à l'heur, j'ai regardé mon film préférer. J'ai commencer à ranger mes vaitements, mes joués, balaier le sol et décorer les mur...

Le message n'est pas évident car il y a une absence de liaison entre les différentes idées formant chaque passage; les auteurs apprenants en sautant d'une idée à l'autre n'ont pas pu assurer la cohésion de leurs textes.

- L'absence de contradiction - Suite à tout ce que nous avons évoqué, un texte doit suivre un certain enchaînement logique, c'est - à - dire il ne doit pas contenir des contradictions ni au niveau du temps, ni au niveau des événements, ni au niveau des personnages. Les deux extraits que nous allons présenter, par la suite, mettent en évidence différentes situations de contradiction :

Passage $1:(\ldots)$ J'ai saisi mon cahier de souvenir entre mes mains. ${ }^{*} \mathrm{Il}$ était propr et bien décorer (...) ${ }^{*}$ ce cahier qui revient à ma troisième année de primair était abîmé et poussiéreux.

Passage 2: Ma grand - mère préparait dans la cuisine les plats pour fêté l'arrivé des nouveau mariés. Pour l'aidé, j'ai ranger les chambre. *J'ai commencer par la terrasse après *rez de chaussée et vers la fin la chambre de ma tante. Dans cette chambre mi-obscur, j'ai trouvé une grande boîte....

Chaque passage véhicule un exemple ou plus de contradiction, comme c'est le cas entre «*Il était propr et bien décorer» et "*ce cahier qui revient à ma troisième année de primair était abîmé et poussiéreux.», et entre «J'ai commencer par la terrasse après » et "*rez de chaussée et vers la fin la chambre de ma tante ».

\section{Erreurs de forme}

Erreurs morphologiques

Erreurs grammaticales
Pour analyser les erreurs portant sur la grammaire, nous avons choisi de travailler selon trois niveaux, à savoir: l'accord, le genre et le temps. Signalons que ce classement d'erreurs grammaticales est basé, d'une part, sur le critère de leur " fréquence » dans les différents d'élèves textes, et, d'autre part, sur notre expérience comme chercheur en didactique des langues et ancien professeur du FLE au cycle secondaire qualifiant.

1. Erreurs affectant l'accord

- Accord Sujet - Verbe

En français, comme dans la plupart des langues vivantes, le verbe s'accorde avec son sujet en prenant les marques de flexion verbale ou appelées autrement «désinences». Une telle règle constitue encore une source de problèmes pour certains élèves en classe du FLE, qui n'arrivent pas à l'appliquer correctement en fonction du contexte demandé. En voici quelques exemples qui illustrent ce propos: «les objets *était rangé », "toute la famille et moi, *était sorti», " ${ }^{*} c^{\prime}$ étaient le cadeau de mon père», «je les ai ${ }^{*}$ chercher partout », et « afin *qu'ils n'oublie_pas ». D'après les exemples ci - dessus, nous remarquons que ces apprenants se trouvent incapables d'accorder le verbe avec son sujet pour deux raisons : d'un côté, leur usage incorrect et erroné de la conjugaison du français, et, de l'autre côté, l'influence de leur langue maternelle, l'arabe dialectal marocain (ADM), une langue qui ne prend pas en considération, par exemple, du sujet dans sa forme «duelle».

\section{- Accord déterminant - Nom}

En français, les déterminants s'accordent sans problème avec le(s) nom(s) qui qualifient en genre et en nombre, règle qui n'est pas respectée dans un certain nombre des copies d'apprenants. Cette méconnaissance de la règle souligne tout simplement l'influence de leur langue maternelle (ADM), qui ne possède pas malheureusement toutes les catégories des déterminants véhiculées en français, en l'occurrence en 
l'occurrence les adjectifs (possessifs, démonstratifs, indéfinis, numéraux), les articles (indéfinis, définis et partitifs) et les adjectifs qualificatifs.

Pour mieux cerner ce point, prenons les exemples suivants: " ${ }^{*}$ Cette objet est abîmé», «*Mon poupée offert par mon grand - père », " ${ }^{*}$ Cet découverte était pour moi un moment inoubliable» et «*Ma grand - mère était un personne aimable et gentil».

2. Erreurs affectant le genre

D'après l'analyse de notre corpus, nous avons constaté qu'un bon nombre d'apprenants a toujours de sérieux problèmes au niveau du genre des mots en français, comme l'illustrent les exemples suivants : «mon frère $\mathrm{m}^{\prime}$ a donné une *vélo pour mon anniversaire», «* ${ }^{*}$ était ma première anniversaire », « En cherchant dans mon petit *chambr», «* mes parents $\mathrm{m}$ 'a offert cette belle abloum» et «Ma grand - mère était un *personne aimable et gentil».

Cette incapacité des apprenants réside en la confusion entre le genre demandé en français et le genre véhiculé en arabe, langue maternelle de la plupart d'entre eux, à l'exemple de :

- En arabe, on dit « شمس šems» (genre féminin); en français, on dit « soleil » (genre masculin).

- En arabe, on dit « كرسي kursi» (genre masculin); en français, on dit « chaise » (genre féminin).

3. Erreurs affectant le temps

- Le passé simple à la place de l'imparfait

Observons les exemples suivants: «Dans la cave, il *fit nuit et il *a eu un clame fatal», «j'*écoutai attentivement ma mère avant de commencer ma tâche», et "quotidiennement, je *sortis de l'école et *accompagnai mon père dans son grand atelier qui était près $d u$ souk de Haïk. Ce jour - là, une grande boîte, qui était posée tout près de la porte, suscitait ma curiosité.»

Dans les deux premiers exemples, Il s'agit d'actions qui durent dans le temps. On est obligé, donc, d'insérer l'imparfait pour exprimer l'aspect duratif des verbes dans le passé.

Dans le troisième exemple, de par l'emploi de l'adverbe «Quotidiennement», l'emploi de l'imparfait devient une exigence pour remplir l'aspect itératif du verbe.

\section{- L'imparfait à la place du passé simple}

Suite à l'examen des exemples suivants : "je remarquais* qu'il avait caché dans sa grande boîte un objet précieux», «je *remarquais qu'il *cachait dans sa grande boîte un objet précieux » et «je *remarquais qu'il *a caché dans sa grande boîte un objet précieux ", nous déduisons que certains apprenants ont conjugué le verbe «remarquer» à l'imparfait à la place du passé simple, et le verbe «cacher » à l'imparfait et au passé composé à la place du plus - que parfait. Avec un tel comportement, ils expriment leur méconnaissance totale de la notion d'aspect du verbe et de la concordance des temps, deux notions temporelles qui prennent en considération la postériorité ou l'antériorité des actions dans un énoncé.

4. Erreurs affectant l'emploi des deux auxiliaires « être » et « avoir »

Dans notre travail, nous nous sommes intéressé à deux aspects d'insuffisance marquant l'emploi des auxiliaires «être » et «avoir»: il s'agit du choix de l'auxiliaire convenable et de sa conjugaison.

Pour le premier, nous avons trouvé les exemples suivants : « $j^{\prime *}$ ai parti chercher le vase de la cave», "je *suis sorti le cahier de souvenir de la grande boite au placar» et « J'*ai descendu au débaras qui se trouvé au sou sol». Cette confusion entre l'emploi de l'auxiliaire «être» ou de l'auxiliaire «avoir» est justifiée essentiellement par l'incapacité des apprenants à identifier, d'après le contexte, le vrai statut des verbes qui les accompagnent, qu'il s'agit d'un verbe d'état ou d'un verbe d'action.

Pour le deuxième, nous avons choisi plusieurs cas présentant la fausse 
manipulation de la conjugaison, et devant lesquelles les apprenants n'arrivent pas à donner la forme correcte de l'auxiliaire « être » ou de l'auxiliaire « avoir ». Citons, par exemple, la confusion entre la forme « est » (l'auxiliaire «être » à la troisième personne du singulier) et la conjonction de coordination « et » (« Il * et rentrer tard ce jour - là.»), et entre la forme «a» (l'auxiliaire «avoir» à la troisième personne $\mathrm{du}$ singulier) et la préposition « à » (« je descendis tout seul *a la cave mi - obscur.»). Ces deux cas de confusion trouvent leur légitimité dans le fait que les deux mots de chaque couple sont pris par les apprenants pour des homophones.

\section{Erreurs lexicales}

D'après Laniel (1991, p.80), les erreurs lexicales désignent toutes les difficultés accompagnant les choix lexicaux des apprenants durant une activité orale ou écrite. De telles difficultés, ajoute Laniel (1991, p.80), sont dûes en grande partie à leur méconnaissance partielle ou totale des propriétés sémantiques de la langue, objet d'apprentissage.

Suite à cette définition et à l'analyse des copies d'élèves collectées, nous avons pu répertorier les erreurs lexicales en deux types essentiels, à savoir les erreurs issues de l'emploi des emprunts et les erreurs issues de la confusion du lexique.

1. Erreurs issues de l'emploi des emprunts

Dans cette sous - classe, nous avons retenu seulement les mots qui sont empruntés de l'arabe, à l'exemple de «mon ancienne chemise * turkouaz», de « Dans un coin cacher de la *cav, j'ai trouver mon ancien *bikchlète qui été ", de « ${ }^{*} c^{\prime}$ était de couleur *blankassé» et de «* j'ai monter à la *tirass de la maison». Nous pourrons dire que les apprenants ne disposent pas d'un arsenal linguistique suffisant pour transmettre de tels messages, cela les pousse à emprunter les mots tels qu'ils sont dans la langue donneuse. Nous pourrons dire également qu'ils ont la conviction que les formes graphiques des mots empruntés sont identiques à celles qui existent dans la langue emprunteuse.
2. Erreurs issues de la confusion du lexique. À ce stade, nous avons repéré deux sous groupes d'erreurs : le premier est lié à la non - identification des phonèmes et le deuxième à la mauvaise distinction auditive.

- La non - identification des morphèmes

Prenons les exemples suivants: «je descendi au * rédechaussé pour chercher * mesafaires du sport », « ${ }^{*}$ cété mon père qui *mavé offert pour *lanniversair 6 ans», et « en cherchant au fend du tiroir, *jé trouvé mon stylo préféré. »

L'analyse de ces exemples nous permet de dire qu'une minorité d'apprenants trouve encore du mal à identifier les phonèmes constituant les mots de la chaîne parlée. Un tel acte provoque, surtout à l'écrit, un emploi incorrect des homophones.

- La distinction auditive

La distinction auditive est une procédure qui sert à aider les élèves à percevoir et reconnaître sans aucun problème les différents sons dans la chaîne parlée. Parfois, certains apprenants, comme le cas dans notre étude, se trouvent incapables de percevoir, voire de distinguer, entre certains phonèmes partageant les mêmes traits distinctifs. Cette attitude les entraîne automatiquement à commettre plus ou moins des erreurs lexicales qualifiées de sérieuses lors de leurs productions écrites.

Pour mieux comprendre ce point, citons le cas de confusion entre «qui » et " qu'il », qui est véhiculé dans les exemples suivants : "à l'ami à *qu'il je faisais tellement confiance », «bien *qui ne soit pas d'accord, mon père n'avait pas changé de position » et «avec de tel geste, je souhaitais * qui soit heureux dans sa vie ». À l'examen de ces exemples, nous remarquons que la plupart des apprenants sont victime de leur prononciation erronée de « qui » et «qu'il», vu qu'ils tendent à prononcer le «qui» en ajoutant le phonème [l], et à prononcer le « qu'il » en supprimant le [1]. 


\section{Erreurs orthographiques}

Les erreurs orthographiques sont présentes dans toutes les copies d'élèves sans exception. Elles sont répertoriées selon deux niveaux d'insuffisance des apprenants, à savoir phonologique et grammatical.

- Issues d'une insuffisance phonologique Sous cet angle, nous n'avons gardé que les cas les plus pertinents:

- L'omission des lettres muettes: à l'écrit, l'apprenant supprime des lettres soit en position médium, comme dans les mots *noisete, ${ }^{*}$ recomençai ${ }^{*}$ finalment et *apris, soit en position finale, comme dans les mots *toujour, *pèr, *objé, *visag. En adoptant un tel comportement, l'apprenant préfère le chemin le plus court, et choisit, ainsi, de simplifier l'orthographe des mots en n'écrivant que les consonnes et les voyelles qu'il entend.

- L'emploi des accents : pour mieux cerner ce propos, prenons les exemples suivants : " ${ }^{*}$ dés ce jour - ${ }^{*} l a »$, «il le trouvait *a la cave» et «ma *poupee était *abimé et *dechirée ». Ces exemples nous ont permis de repérer deux fausses attitudes adoptées par la majorité des apprenants. Par la première attitude, ils mettent les accents d'une façon hasardeuse, c'est-à-dire ils ne suivent aucune règle pour effectuer une telle tâche (ex. *dés). Par la deuxième attitude, ils oublient totalement ou partiellement des accents ou des apostrophes; cette absence des accents (ex. *a, *abimé, * poupee, *dechirée ) et / ou de l'apostrophe, est expliquée par l'influence de l'apprentissage de l'anglais, qui est une langue étrangère sans accent. Il est à signaler qu'aucun apprenant n'a été exclu de cette rubrique, mais la fréquence de ce type d'erreur dans les copies a varié selon les compétences linguistiques de chacun d'eux.

- Issues d'une insuffisance grammaticale Analysons de près les exemples suivants : « je l'ai * trouver jauni et abîmé » et « ils * été heureux de me voir dans de telle situation.»

Dans l'exemple (1), l'apprenant n'a pas bien conjugué le verbe "trouver ", c'est à - dire il n'a pas trouvé la forme idéale à un ce contexte (Participe passé conjugué avec l'auxiliaire « avoir »). Pour l'exemple (2), il a mal accordé le verbe "être » avec son propre sujet, et, il a gardé, par conséquent, la même forme aussi bien au singulier qu'au pluriel.

\section{Erreurs syntaxiques}

Il sera question, dans cette catégorie, des erreurs syntaxiques qui affectent particulièrement l'ordre des mots dans les différentes phrases d'un texte, à l'exemple de la place de l'adjectif qualificatif et de la répétition.

Concernant la place de l'adjectif qualificatif, nous avons pu dévoiler plusieurs cas dans lesquels les apprenants transgressent la ou les règle(s) déjà prescrite(s) dans la grammaire française. Dans le tableau suivant, nous présentons les règles et quelques exemples erronés :

Tableau 2. La place de l'adjectif qualificatif entre la règle à appliquer et les cas erronés

\begin{tabular}{|c|c|}
\hline Règles & Exemples erronés \\
\hline $\begin{array}{l}\text { - l'adjectif monosyllabique qualifiant un } \\
\text { polysyllabique est toujours antéposé. } \\
\text { - L'adjectif polysyllabique qualifiant un nom } \\
\text { monosyllabique est postposé. } \\
\text { - L'épithète accompagnée d'un adverbe est } \\
\text { généralement postposée. } \\
\text { - L'adjectif antéposé a tendance à se souder au nom. } \\
\text { L'adjectif postposé conserve son accent propre, il garde } \\
\text { son autonomie, et souligne une qualité distinctive de ce } \\
\text { dont on parle. }\end{array}$ & $\begin{array}{l}\text { «C'était mon ouvrage préféré, une grammaire } \\
\text { *bonne. » (1) } \\
\text { «Dans lequel mon ami m'écrit un *harmonieux } \\
\text { vers. » (2) } \\
\text { «Ce licol appartenant à un cheval *vigoureux. } \\
\text { remarquablement» (3) } \\
\text { « Mon grand - père qui était un homme *grand par } \\
\text { son intelligence» }(4)\end{array}$ \\
\hline
\end{tabular}


D'après ces exemples erronés, nous avons pu souligner l'incapacité de certains apprenants à appliquer la ou les règle(s) régissant l'emplacement adéquat des adjectifs qualificatifs, surtout à fonction épithète, par rapport au nom qu'ils qualifient. Cet emplacement est régi généralement par trois règles, à savoir la règle rythmique (le nombre de syllabe de l'adjectif et du nom qualifié) (voir les exemples 1 et 2), la règle syntaxique (l'emplacement de l'adjectif dépend aussi de l'emplacement des autres éléments dans la phrase) (voir l'exemple 3) et la règle sémantique (on prend en considération les différents sens de l'adjectif, sens propre et / ou sens figuré) (voir l'exemple 4).

À propos de la répétition, nous avons $\mathrm{pu}$ dégager un bon nombre d'exemples illustrant ce propos, comme «mon père * il m'a offert ce bijou précieu», « je *1'ai remercié ma mère pour ce belle cadeau», « *moi j'était seul» et " $j^{\prime *} y$ monté sur la terrésse pour cherché dans le paqué». De tels exemples nous ont laissé devant un type d'erreurs qui peut affecter la phrase, pas seulement au niveau syntaxique mais aussi aux niveaux sémantique, lexical et morphologique. Il nous apparaît ici que les apprenants commettent de la répétition soit c'est par faute d'inattention, soit c'est par incompétence et méconnaissance de la ou les règle(s) à appliquer.

\section{Remédiation}

Dans cette partie, notre but sera de suggérer quelques démarches que peuvent adopter les enseignants et leurs apprenants en classe du FLE afin de faire face à toutes les erreurs rencontrées durant l'enseignement / apprentissage de la production écrite, de trouver leurs sources et d'y remédier, par la suite.

\section{Concernant les enseignants}

En ce qui concerne les démarches à effectuer afin de corriger toute erreur et $y$ remédier, les enseignants devraient consister essentiellement à libérer ses apprenants de toute charge psychologique négative en les persuadant que l'erreur, comme étant une partie intégrante $\mathrm{du}$ processus d'apprentissage du FLE, est inéluctable mais corrigible. Un tel comportement positif et optimiste est toujours appuyé par la motivation qui suscite l'engagement total ou partiel de l'apprenant à «accomplir l'activité d'apprentissage qu'on lui propose et de persévérer dans l'accomplissement de celle-ci et ce, dans le but d'apprendre » (Viau, 2009, p.12).

De plus, l'enseignant aurait également la possibilité de choisir entre différentes pistes selon le point de vue duquel la remédiation sera abordée, c'est - à - dire selon le niveau de l'apprenant, le type de l'erreur et la démarche d'apprentissage. Ainsi, d'après Lyster (2001, p.272), le professeur peut aborder l'erreur accompagnant l'apprentissage d'une langue, aussi bien à l'oral qu'à l'écrit, de trois manières: la première consiste à corriger directement l'erreur qui représente la réponse incorrecte de l'élève; la deuxième s'explique par la correction implicite de l'erreur en proposant des exemples pareils à la réponse de l'élève, mais qu'ils sont corrects; de ce fait, il fera des comparaisons et déduira inconsciemment ses erreurs; et, la troisième met l'accent sur une correction par négociation entre l'enseignant et l'apprenant jusqu'à ce qu'ils se mettent d'accord sur la bonne réponse.

Selon Bhatia (1974, p.347), l'enseignant doit classer les différentes erreurs collectées lors d'une activité écrite ou orale en fonction du seul critère de "fréquence». Autrement dit, l'enseignant doit, tout d'abord, repérer, analyser et corriger les erreurs qui sont les plus fréquentes en classe, et qui demandent, selon Bhatia, une correction en groupe; après, en deuxième position, viennent les erreurs les moins fréquentes, et qui exigent un traitement individuel ciblant chaque apprenant à part.

Contrairement aux deux premiers didacticiens, Wingfield (1975, p.321-326) n'a pas donné de l'importance au type de l'erreur dégagé durant une activité quelconque (plus fréquent / moins fréquent ou plus grave / moins grave), mais il a plutôt mis l'accent sur la position que peut prendre l'apprenant lors d'une séance de correction. En première position, l'apprenant est actif, et 
il est amené, ainsi, à l'autocorrection, une situation devant laquelle il se sentira explicitement impliqué dans le traitement des erreurs et sera amené à y réfléchir afin de les réduire au minimum. En deuxième position, l'apprenant est passif et est obligé de retenir les erreurs soulevées par l'enseignant soit à l'oral soit à l'écrit.

D'ailleurs, l'enseignant devrait aussi choisir ses exercices de remédiation selon la nature de l'erreur et également le niveau et l'habileté de ses apprenants, par exemple si les élèves ont encore des insuffisances au niveau de l'«accord des adjectifs», l'enseignant, après leur avoir rappelé les règles de l' "accord » enseignée(s), peut prononcer des groupes de mots, et les élèves sont invités à écrire ce qu'ils entendent. Son travail se réalise d'une façon progressive dans la mesure où il peut aborder avec ses élèves le genre des adjectifs puis le nombre, avant d'aborder les adjectifs de couleur. Un autre exemple pour illustrer ce propos apparait lorsque les apprenants font des choix lexicaux déplacés; dans ce cas, l'enseignant, pour une remédiation efficace, doit les inciter à manipuler les dictionnaires surtout unilingues en leur proposant une série de phrases ou de textes avec des vides à remplir.

\section{Concernant les apprenants}

Dans cette partie, nous avançons quelques démarches que les apprenants peuvent suivre afin qu'ils mettent fin à toutes les erreurs qui accompagnent leur apprentissage de l'écrit, les corriger et y remédier sans aucun problème. Parmi ces démarches, nous trouvons: Premièrement, nous recommandons à l'apprenant de bien respecter toutes les propriétés marquant chaque étape du processus rédactionnel, quelle que soit la durée et la forme de la production écrite réalisée; par exemple, pour la planification, il est obligé de dégager les mots - clés, créer les idées et les organiser au moyen d'un plan bien précis.

Deuxièmement, nous conseillons aux apprenants d'être toujours les premiers lecteurs de leurs propres textes. Une lecture et relecture permettront d'apporter à leur texte une correction à tous les niveaux, à la forme et au contenu. Pour compléter cette tâche, l'apprenant, à l'aide de leur enseignant, doit, avant tout, opérer à une autocorrection des différentes erreurs repérées dans son propre écrit. Cette autocorrection peut prendre une autre forme évolutive et, devenir, ainsi, une co-correction qui suscite l'intervention de tous les apprenants de la classe, dans le cadre d'un échange fructueux, pour en discuter et découvrir la ou les règle(s) adéquate(s) pour y remédier. Il est fort possible, dans une telle démarche, que l'enseignant permet sans aucun problème à l'apprenant d'utiliser un dictionnaire (français - français) pour chercher ses mots et en vérifier l'orthographe et la conjugaison.

Troisièmement, les apprenants doivent effectuer en dehors de la classe des lectures des textes en français de tous les genres et de tous les types, à savoir des romans, des poèmes, des pièces de théâtre, des articles, des contes et des essais, pour qu'ils puissent éviter toute interférence avec les autres langues, améliorer tous les aspects de la langue, objet d'apprentissage, et les perfectionner.

\section{CONCLUSION}

En guise de conclusion, l'analyse des productions écrites des apprenants en classe du FLE, à l'exemple de la deuxième année baccalauréat toutes filières confondues, nous a permis de classer les erreurs repérées en deux grandes catégories, les erreurs de contenu, qui affectent la cohérence et la cohésion du texte, et les erreurs de forme, qui affectent la langue du texte à tous les niveaux morphologiques, lexicaux, orthographiques et syntaxiques. Une telle catégorisation des erreurs à l'écrit a trouvé sa légitimité tantôt dans les incapacités et les méconnaissances linguistiques des apprenants, tantôt dans les influences de leurs langues maternelles (l'arabe avec ses variétés ou l'amazighe avec ses différents parlers) et tantôt dans les langues étrangères composant son entourage linguistique.

Dans la dernière partie de notre étude, nous avons proposé quelques remédiations aux erreurs de l'écrit qui permettront non seulement d'identifier les niveaux de 
compétences littéraires, linguistiques et communicatives des apprenants mais aussi de préciser les problèmes rencontrés afin d'avancer, tout d'abord, tous les paramètres convenables lors du parcours d'apprentissage de la production écrite en suivant les objectifs fixés à l'avance, et, ensuite, de mettre au point de nouvelles démarches et approches pédagogiques convenables aux différents besoins scripturaux des apprenants.

\section{REMERCIEMENTS}

Je tiens à exprimer mes vifs remerciements à la direction de la Faculté Polydisciplinaire d'Errachidia (FPE), au nom de son Doyen M. Majidi LHOU, pour l'appui financier et le soutien moral inestimable qu'elle $\mathrm{m}$ 'a apportés pour la réalisation de ce travail.

Je témoigne ma profonde reconnaissance aux directrices et directeurs des établissements visités: Mme Naïma OUZAHRA, M. El-arbi EL-KARKARI, M. Ahmed EL-ABDALAOUI et M. Mohammed OUMIRI. Grâce à leur aide précieuse, j’ai pu mener à bien ma recherche et aboutir à des résultats très satisfaisants.

Mes remerciements vont également à toutes les personnes qui ont contribué de près ou de loin à la réalisation de cette étude, notamment les enseignants de la langue française qui n'ont épargné aucun effort pour m'aider tout au long de mon enquête de terrain.

\section{RÉFÉRENCES}

Bhatia, A. T. (1974). An error analysis of students' compositions. IRAL, 12(4), 337-350.

Caroline, H-T. (2014). L'apprentissage $d u$ français langue seconde : les erreurs interlangues chez des élèves en classe d'immersion précoce au Nouveau Brunswick. (Mémoire de master 2 professionnel en sciences du Langage). Université Stendal 3, Grenoble.

Laniel, D. (2005). Le vocabulaire en français langue seconde : de 'parent pauvre' de l'enseignement à 'invité d'honneur' des communications assistées par ordinateur. Revue de l'Association Québécoise des Enseignants de Français Langue Seconde, 25(2), 73-100.

Lennon, P. (1991). Error: some problems of definition and identification. Applied Linguistic, 12(2), 180-195.

Lyster, R. (2001). Negotiation of form, recasts, and explicit correction in relation to error types and learner repair in immersion classrooms. Language Learing, 51, 265 - 301.

Viau, R. (2009). La motivation en contexte scolaire. Bruxelles : De Boeck.

Wingfield, R. J. (1975). Five ways of dealing with errors in written composition. English Language Teaching Journal, 29 (4), 311-313. 